\title{
Security System Based on Ultrasonic Sensor Technology
}

\author{
Shinu N Yoannan ${ }^{1}$, Vince T Vaipicherry ${ }^{2}$, Don K Thankachan ${ }^{3}$ \\ Prof. Ram Prasad Tripathy ${ }^{4}$ \\ ${ }_{1,2,3}$ (M.Tech. Scholar, Applied Electronics and Instrumentation, LMCST, University of Kerala, India) \\ ${ }^{4}$ (Prof., Department of Electronics and Communication Engineering, LMCST, University of Kerala, India)
}

\begin{abstract}
In this paper we design and implement a security system with an ultrasonic sensor module to enhance the system's reliability. The ultrasonic sensor contains a transmitter and a receiver and the module is placed in a rotating motor. It is assumed that an ultrasonic sensor is set in a rotating motor to cover a wide range. The Ultrasonic transmitter periodically emits ultrasonic signals into an open area. A rotating motor is used to allow the sensor to cover whole 360 degrees. If the signal ever hits any physical objects, it will be reflected back and the receiver part of the sensor will then capture it. The microcontroller unit (MCU) will constantly check for the receiver output of the ultrasonic transmitter. If the receiver output is high, the MCU will perform distance analysis of the object from the sensor using the fact that ultrasonic waves travel in air at $340 \mathrm{~m} / \mathrm{s}$. The time taken for the waves to hit the object and return can be calculated as the time taken for the receiver output to be high after the transmitter has been initiated to send ultrasonic waves. Once the distance is calculated, MCU checks whether the object is within the range threshold specified within the MCU for initiating the alert. If the object is within the range threshold, the MCU initiates a sound alarm and also the global system for mobile communications (GSM) modem to send short message service (SMS) or call to the concerned person.
\end{abstract}

Keywords: GSM Module,Microcontroller unit(MCU),Motor controller driver unit,Ultrasonic sensor(obstacles detection).

\section{INTRODUCTION}

We were inspired to build a Security system based on ultrasonic sensor technology. Security is an important part of home, banks, shops and so on. The traditional security systems based on opening of doors and windows. Our ultrasonic sensor technology is used, it detects the intruders on their physical presence. An ultrasonic sensor can measure the distance to an obstacle within its conic beam. The AVR family microcontroller was used to interface with the ultrasonic sensor. Here we used Atmega16A microcontroller. The key features of Atmega16A microcontroller that we used for this paper are the three timers and the serial rs 232 USART. The timers are responsible of scheduling periodical execution of various tasks, generate alarm tone, and generate PWM signal with appropriate duty cycle and frequency to control the motor. Also, use the motor drivers and it acts as current amplifiers since they take a low current control signal and provide a higher current signal. The range of the ultrasonic sensor can be changed by using an external adjustment device. Fig.1 shows the logical structure of Security system based on ultrasonic sensor technology.

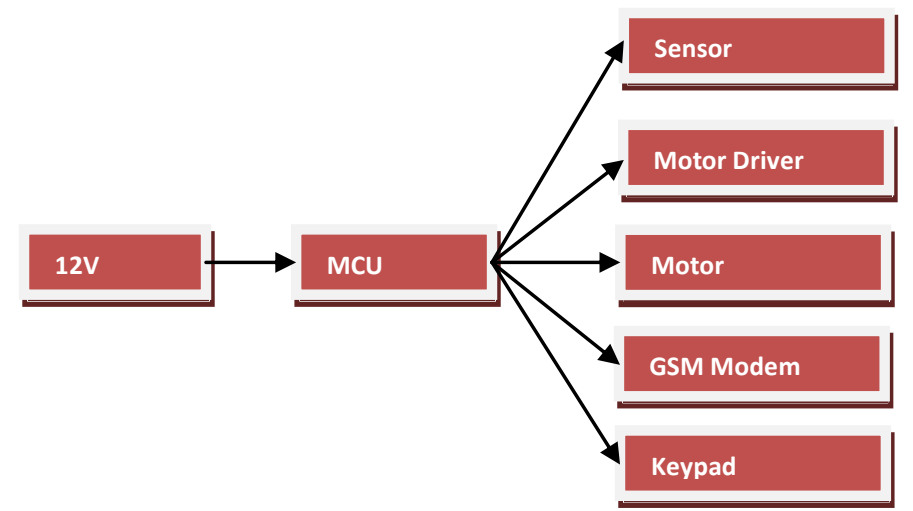

Fig. 1 shows the logical structure

\section{PRINCIPLES}

Figure 1 shows a logical structure of the system. The security system consists of one ultrasonic sensor, a microcontroller, a motor driver controller, GSM module and a DC motor. The system uses ultrasonic sensors that has a transmitter part and receiver part. The ultrasonic transmitter periodically emits ultrasonic signals into 
an open area in front of it. To cover a wide range, a rotating motor is used to allow the sensor (transmitter and receiver pair) to cover roughly 180 or 360 degrees according to our need. If the signal ever hits a physical object, it will be reflected back and, the receiver part of the sensor will then capture it with the object considered detected as its position (distance from the device and angle relative to the device) is now known.

\section{A. Ultrasonic Sensor}

\section{DESCRIPTION}

The principle of working of an ultrasonic sensor is easy. The sensor transmits ultrasonic sound waves and waits for reflected sound waves. After receiving reflected sound wave or usually named echo, sensor detects the distance in different ways. Fig.2 shows the detection of an ultrasonic sound waves in an object. Ultrasonic ranging module $\mathrm{HC}-\mathrm{SRO} 4$ provides $2 \mathrm{~cm}-500 \mathrm{~cm}$ non-contact measurement function, the ranging accuracy can reach to $3 \mathrm{~mm}$. The modules includes ultrasonic transmitters, receivers and control unit. The ranges can be increased by using other type of ultrasonic sensors. The basic principle of work:

- Using IO trigger for at least 10us high level signal.

- The Module automatically sends eight $40 \mathrm{kHz}$ and detect whether there is a pulse signal back.

- If the signal back, through high level, time of high output IO duration is the time from sending ultrasonic to returning.

- $\quad$ Test Distance $=($ high level $*$ velocity of sound $) / 2$.

It has stable performance and high ranging accuracy and make it a popular module in electronic market. Compared to the shape IR ranging module, it is more inexpensive. But it has the same ranging accuracy and longer ranging distance. There are 4 pins out of the module: VCC, Trig, Echo, GND. So its a very easy interface for controller to use ranging. The all process is : pull the Trig pin to high level for more than 10us impulse, the module start ranging; finish ranging, if you find an object in front, Echo pin will be high level, and based on the different distance, it will take the difference of high level. So we can calculate the distance easily.

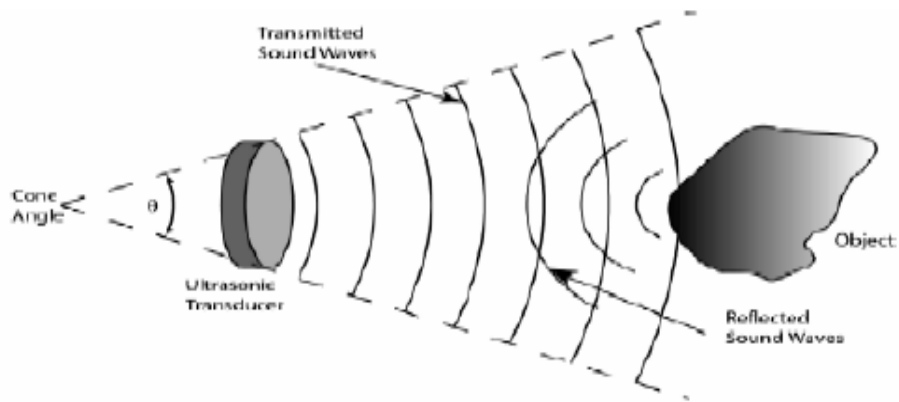

Fig. 2 shows the detection of an ultrasonic sound waves in an object.

Figure 3 shows the timing diagram after triggering sensor with a TTL logic 1 (we use $5 \mathrm{~V}$ ) at least 10us, sonic burst module embedded in sensor makes eight cycle sonic burst at $40 \mathrm{kHz}$. After the last sonic burst go low our echo signal became high and stay high until any reflected sound received by the sensor.

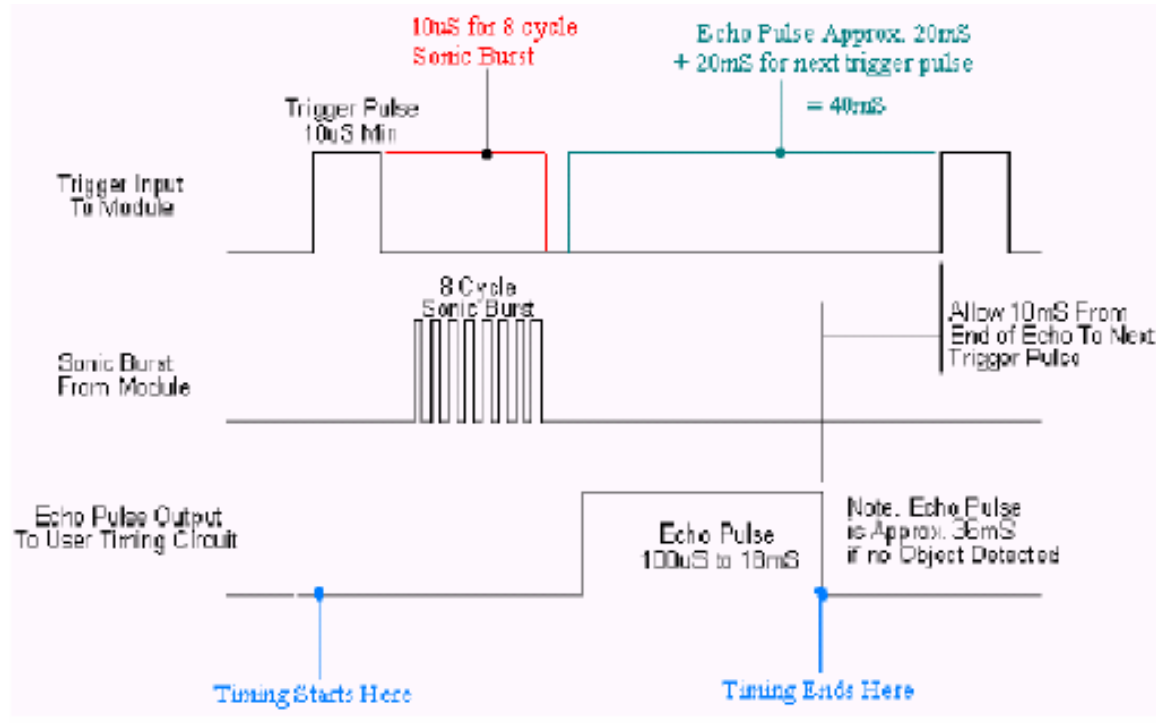


Fig.3 shoes the Timing Diagram after triggering sensor with a TTL logic at least 10us.

\section{B. Microcontroller Unit}

Microcontroller can be termed as a single on chip computer which includes number of peripherals like RAM, EEPROM, Timers etc., required to perform some predefined task. There are number of popular families of microcontrollers which are used in different applications as per their capability and feasibility to perform the desired task, most common of these are 8051, AVR and PIC microcontrollers. To increase the system performance we use AVR family microcontroller. Here, use Atmega16A microcontroller. It consists three timers and the serial rs232 USART. The serial USART is used to communicate with the ultrasonic sensor and the modem. The Atmega16A is a low cost and feature rich AVR microcontroller from ATMEL. The main advantage is, it contains JTAG interface. Thus it is extensive On-chip Debug support. Programming of Flash, EEPROM, Fuses, and Lock Bits through the JTAG interface. The program(Embedded C) can be stored in the chip and according to that the system can perform.

\section{GSM Module Unit}

The GSM Module Unit is able to send and read SMS or call, connect to internet via general packet radio service (GPRS) through simple AT commands. The modem needed only three wires (Tx, Rx, GND) except power supply to interface with microcontroller unit. Here we use SIM900A.

\section{Motor Controller Unit}

The motor controller driver unit is dual H-bridge motor driver integrated circuit(IC). It acts as current amplifier since they take a low-current control signal and provide a higher-current signal. This higher current signals is used to drive the motors. Here use L293D motor driver IC. It is used to change the direction and to adjust the speed of the motor. Thus the motor will rotate in clockwise and anticlockwise directions. When an enable input is high, the associated driver gets enabled. As a result, the outputs become active and work in phase with their inputs. Similarly, when the enable input is low, that driver is disabled, and their outputs are off and in the high-impedance state.

\section{Implementation Results}

\section{A. Distance measurement accuracy}

Our sensor calibration and utilization gave us a distance measurement with roughly $1.5 \mathrm{~cm}$ accuracy. Provided that we are using the sensor to detect presence of an object more so than the exact distance, this accuracy is tolerable.

\section{TABLE I}

TEST RANGE AND DETECTION MEASURED TABLE

\begin{tabular}{|c|c|c|}
\hline Test Range & Measured Detection Distance & Echo Output High Time \\
\hline 1 & $7.5 \mathrm{~cm}$ & $500 \mathrm{us}$ \\
\hline 2 & $15 \mathrm{~cm}$ & $1000 \mathrm{us}$ \\
\hline 3 & $30 \mathrm{~cm}$ & $2000 \mathrm{us}$ \\
\hline 4 & $60 \mathrm{~cm}$ & $4000 \mathrm{us}$ \\
\hline 5 & $100 \mathrm{~cm}$ & $6600 \mathrm{us}$ \\
\hline
\end{tabular}

\section{B. Speed of detection}

It takes a maximum of three seconds for the system to detect a newly presented object, and to turn on the alarm. This is because the motor is set to cover 180 or 360 degree range, and thus it takes roughly 3 seconds for it to start from one angle and back. However, a user can easily configure the motor to a narrower angle to focus at specific area(such as doorway), in which case the sensor will detect newly present object in less than a second.

\section{Detection accuracy}

Through this paper, we believe our system is capable of detecting intruders $95 \%$ of the time, provided that sensors was placed at appropriate position. The few times that intruder get away are when they are capable of crossing past the sensor quicker than 200 millisecond, which is our measurement interval hard-coded into our code.

\section{Message notification}

Upon object detection, we successfully receive SMS or call that contain the exact time when the security was triggered. This is a standard delivery, and therefore it could take a minute or two for it to reach the concerned cell. When the object continues to be in the way, the system does continue to send message or call 
according to the program set. So far, we have not seen any false positive alerts. Table II shows the primary results of our experiments.

TABLE II

IMPLEMENTATION RESULTS OF DIFFERENT SENSING DISTANCES

\begin{tabular}{|c|c|}
\hline Distance & Overall sensing probability \\
\hline $1 \mathrm{~m}$ & $100 \%$ \\
\hline $2 \mathrm{~m}$ & $99 \%$ \\
\hline $3 \mathrm{~m}$ & $94 \%$ \\
\hline $4 \mathrm{~m}$ & $87 \%$ \\
\hline $5 \mathrm{~m}$ & $79 \%$ \\
\hline
\end{tabular}

\section{CONCLUSION}

We have presented a portable security system through this paper. We believe our design possesses competitive features to many of the security system out there, except at much more portable level. However, the accuracy can be increased by using multiple of ultrasonic sensors.

\section{References}

[1]. Sungbok Kim and Hyunbin Kim, "Simple and Complex Obstacle Detection Using an Overlapped Ultrasonic Sensor Ring,"2012 $12^{\text {th }}$ International Conference on Control, Automation and Systems.

[2]. Jun Hou, Chengdong Wu, Zhongjia Yuan, Jiyuan Tan, Qiaoqiao Wang and Yun Zhou, "Research of Intelligent Home Security Surveillance System Based on ZigBee," International Symposium on Intelligent Information Technology Application Workshops,pp.554-557,21-22 Dec.2008

[3]. J. Borenstein, H. R. Everett, and L. Feng, “Where Am I?": Sensors and Methods for Mobile Robot Positioning, The University of Michigan, 1996.

[4] J. L. Crowley, "World Modelling and Position Estimation for a Mobile Robot Using Ultrasonic Ranging,"Proc. IEEE Int. Conf. Robotics and Automation, pp. 674-680,1989.

[5]. H . Choset, K. Nagatani, and N. A. Lazar, "The Arc Traversal Median Algorithm: a Geometric Approach to Increase Ultrasonic Sensor Azimuth Accuracy," IEEE Trans. Robotics and Automation, vol. 19, no. 3, pp. 513-522, 2003.

[6] Ying-Wen Bai, Li-Sih Shen and Zong-Han Li "Design and Implementation of an Embedded Home Surveillance System by Use of Multiple Ultrasonic Sensor".

[7]. Referenced Websites of en.wikiprdia.org/wiki/Ultrasonic Sensor, www.senix.com/Ultrasonic-Sensors

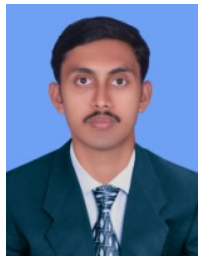

\section{BIOGRAPHY}

Shinu N Yohannan received the B.Tech degree in Electronics and Communication Engineering from Musaliar College of Engineering and Technology, Pathanamthitta, Kerala India, in 2012. He is currently pursuing M.Tech. degree in Applied Electronics and Instrumentation in Lourdes Matha College of Science and Technology, Thiruvananthapuram, Kerala, India.

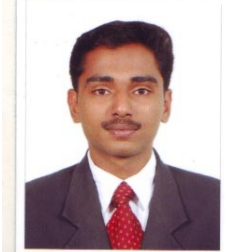

Vince T Vaippicherry received the B.Tech degree in Electronics and Communication Engineering from College of Engineering, Kidagoor, Kerala India, in 2012. He is currently pursuing M.Tech. degree in Applied Electronics and Instrumentation in Lourdes Matha College of Science and Technology, Thiruvananthapuram, Kerala, India.

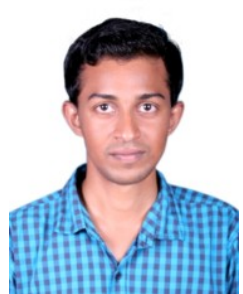

Don K Thankachan received the B.Tech degree in Electronics and Instrumentation Engineering from College of Engineering, Kidagoor, Kerala India, in 2010. From 2010 to 2012, he was with the Cognizant Technology Solutions, Chennai, India. He is currently pursuing M.Tech. degree in Applied Electronics and Instrumentation in Lourdes Matha College of Science and Technology, Thiruvananthapuram, Kerala, India. 